\title{
The structure of the factor group of the unrestricted sum by the restricted sum of Abelian groups II
}

\section{K. Golema and A. Hulanicki (Wroclaw)}

This paper is the continuation of a paper of one of the authors [1] devoted to the description of the structure of the factor group of the unrestricted direct sum $S^{*}$ of countably many Abelian groups $G_{1}, G_{2}, \ldots$ by the restricted direct sum $S$ of these groups.

In [1] is shown that $S^{*} / S$ is algebraically compact and as such it can be characterized by a sequence of cardinals. Since only countable sequences of the groups $G_{1}, G_{2}, \ldots$ are considered, no non-trivial evaluations of the cardinal in question can be expected if the cardinals of the $G_{k}$ 's are allowed to be arbitrary. Clearly enough a reasonable restriction on the cardinals of $G_{k}, k=1,2, \ldots$, is $G_{k} \leqslant 2^{\aleph_{0}}$ for any $k=1,2, \ldots$ Under this assumption the authors are able to show that there are just two possible values for any cardinal in question, namely 0 and $2^{\text {No }_{0}}$. The problem which of the sequences of cardinals, each being 0 or $2^{\mathrm{N}_{0}}$, are the ones prescribed to the group $S^{*} / \mathcal{S}$ finds also its simple answer here.

Algebraically compact groups. The notion of algebraically compact groups is due to I. Kaplansky [3] and is the following.

A group $G$ is algebraically compact if it is of the form

$$
G=D+\sum_{p}^{*} D_{p}
$$

where $D$ is a divisible group and $\sum_{p}^{*} D_{p}$ is the unrestricted direct sum of the groups $D_{p}$, where for a fixed prime $p$ the group $D_{p}$ is the completion in the $p$-adic topology of the restricted direct sum of oyclic $p$-groups and groups of $p$-adic integers.

According to the definition, each of the groups $D_{p}$ is uniquely defined by a subgroup $B_{p}$ (called a basic submodule of $D_{p}$ ) which is the direct sum of, say, $\gamma_{p}^{(n)}$ cyclic groups of order $p^{n}, n=1,2, \ldots$, and $\gamma_{p}^{(0)}$ groups of $p$-adic integers and the completion of which in the $p$-adic topology 
of it is $D_{p}$. As is shown by Kulikoff [4], p. 309, any two such subgroups $B_{p}$ of a fixed group $D_{p}$ are isomorphic, so there is a one-to-one correspondence between the group $D_{p}$ and the cardinals $\gamma_{p}^{(n)}(n=0,1,2, \ldots)$.

Thus the whole group $\sum_{p}^{*} D_{p}$ is defined by the countable famils of cardinals $\gamma_{p}^{(n)}, n=0,1,2, \ldots, p=2,3,5, \ldots$

As to the divisible group $D$, it is the direct sum of some, say, $\delta$ rational groups and of some, say, $\delta_{p}$ Prüfer's groups $C_{p \infty}, p=2,3,5, \ldots$ and, moreover, any two such representations are isomorphic. This means that the cardinals $\delta$ and $\delta_{p}, p=2,3,5, \ldots$, are in a one-to-one correspondence with the group $D$.

Thus we see that any algebraically compact group is fully characterized by the countably many cardinals

$$
\delta, \delta_{p}, \gamma_{p}^{(n)} \quad(n=0,1,2, \ldots, p=2,3,5, \ldots) .
$$

To indicate the dependence of the cardinals on the group $G$ we shall write $\delta=\delta(G), \delta_{p}=\delta_{p}(G), \gamma_{p}^{(n)}=\gamma_{p}^{(n)}(G),(n=0,1,2, \ldots, p=2,3,5, \ldots)$.

The notion of algebraically compact groups was introduced by I. Kaplansky in order to characterize the class of compact (topological) groups. He proved that compact groups are algebraically compact and he proposed to find the conditions on cardinals (2) for which the group $G$ defined by the procedure described above is compact. Although the problem of the structure of compact groups has found its solution in a different way the idea of characterizing of some classes of compact groups by means of cardinals (2) has been exploited by one of the author (see [2]). Here this idea turns out to be fruitful in a quite different circle of problems.

Some lemmas and reductions. We now quote two results of Kulikoff which link more closely the cardinals $\gamma_{p}^{(0)}$ and $\gamma_{p}^{(n)}(n=1,2, \ldots)$ with the group $D_{p}$.

i. (Kulikoff [4], p. 308). The cardinal $\gamma_{n}^{(0)}$ is the number of the direct summands in any decomposition into the direct sum of cyclic groups of order $p$ of the group $D_{p} / g p\{H, p D p\}$, where $\operatorname{gp}\{H, p D p\}$ denotes the group generated by the maximal periodic subgroup $H$ of $D_{p}$ and the group $p D p$.

ii. (Kulikoff [4], p. 308). The cardinal $\gamma_{p}^{(n)}$ with $n \geqslant 1$, is the number of the direct summands in any decomposition into the direct sum of cyctic groups of order $p$ of the group $\left(p^{n-1} D p\right)[p] /\left(p^{n} D_{p}\right)[p]\left(^{1}\right)$.

By these two results the dependence of the cardinals on the group $G$ becomes much simpler.

In fact,

i'. The cardinal $\gamma_{p}^{(0)}$ is the number of the direct summands in any decomposition into the direct sum of cyctic groups of order $p$ of the group $G / \mathrm{gp}\{H, \mathrm{~h} G\}$, where $H$ denotes the maximal periodic subgroup of the group $G$.

(1) For any group $G$, by $G[p]$ we denote the subgroup of elements of order $p$. ii'. The cardinal $\gamma_{p}^{(n)}$ with $n \geqslant 1$ is the number of the direct summands in any decomposition into the direat sum of cyctic groups of order $p$ of the group $\left(p^{n-1} G\right)[p] /\left(p^{n} G\right)[p]$ (cf. $\left.\left(^{1}\right)\right)$.

The proofs of $\mathrm{i}^{\prime}$ and $\mathrm{ii}^{\prime}$ are almost immediate. We simply note that since each of the $D p$ 's with $p \neq p_{0}$ is divisible by any power of $p_{0}$ (cf. [3], p. 50), we have

$$
p_{0}^{n} G=D+\sum_{p \neq p}^{*} D_{p}+p_{0}^{n} D_{p} \quad \text { for any } \quad u=1,2, \ldots
$$

Moreover, if $H$ is the maximal periodic subgroup of $G$, then, since $D_{p}$ does not contain any element of order $s$ prime to $p$,

$$
H=(D \cap H)+\sum_{p}\left(D_{p} \cap H\right)=(D \cap H)+\sum_{p} H_{p},
$$

where $\sum_{p} H_{p}$ is the direct sum of the maximal periodic subgroups $H_{p}$ of the groups $D_{p}$, respectively.

So, by (3) with $n=1$,

$$
\operatorname{gp}\left\{H, p_{0} G\right\}=D+\sum_{p \neq p 0}^{*} D_{p}+\operatorname{gp}\left\{H_{p}, p_{0} D p_{0}\right\} \text {. }
$$

Consequently,

and

$$
G / \mathrm{gp}\{H, p G\}=D_{p} / \mathrm{gp}\left\{H_{p}, p D_{p}\right\}
$$

$$
\left(p^{n-1} G\right)[p] /\left(p^{n} G\right)[p]=\left(p^{n-1} D_{p}\right)[p] /\left(p^{n} D_{p}\right)[p] .
$$

Theorems. From now on we consider an arbitrary but fixed sequence

$$
G_{1}, G_{2}, \ldots
$$

of groups with $\overline{\overline{G_{k}}} \leqslant 2^{\mathrm{N}_{0}}, k=1,2, \ldots$, and we define the unrestricted direct sum $S^{*}$ of $G_{l i}, k=1,2, \ldots$, as the set of the sequences

$$
S^{*}=\left\{\left(x_{k}\right)_{k=1,2, \ldots}: x_{k} \in G_{k}\right\}
$$

and the (restricted) direct sum $S$ of the groups $G_{k}, k=1,2, \ldots$, as the subgroup of $S^{*}$ consisting of the sequences the terms of which are zero from a point onwards. We denote by $G$ the group $S^{*} / S$ and by $\pi$ the natural homomorphism

$$
\pi: S^{*} \stackrel{\text { onto }}{\rightarrow} S^{*} / S=G
$$

The group $G$ is, by [1], algebraically compact, so it is of the form (1). Let $(2)$ be the family of eardinals prescribed to it. 
THEOREM 1. Each of the cardinals (2) is either 0 or $2^{\text {Noo }}$. Moreover, the following relations hold

(a) $\gamma_{p}^{(0)} \geqslant \limsup _{n \rightarrow \infty} \gamma_{p}^{(n)}$

(b) $\delta_{p} \geqslant \limsup _{n \rightarrow \infty} \gamma_{p}^{(n)}$

(c) If $\delta=0$, then

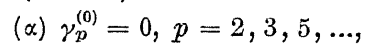

(ß) $\delta_{p}=0, p=2,3,5, \ldots$,

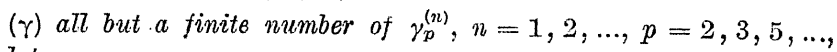
are equal to zero.

Proof. Since $\overline{\overline{G_{k}}} \leqslant 2^{\mathrm{N}_{0}}, k=1,2, \ldots$, we have $\overline{\bar{G}}=2^{\mathrm{N}_{0}}$. Therefore instead of proving that a cardinal of (2) is equal to $2^{\mathrm{No}_{0}}$ it is sufficient to show that it is not less than $2^{\text {No }}$.

We start with the $\gamma_{p}^{(n)}, n=0,1,2, \ldots$ We have to show that, for any prime $p$ and any non-negative natural number $n, \gamma^{(n)}$ is either 0 or $2^{\text {No }_{0}}$ $B y i^{\prime}$ and ii' this is equivalent to the fact that each of the groups $G / g p\{H, p G\}$ and $\left(p^{n-1} G\right)[p] /\left(p^{n} G\right)[p]$ either is trivial or it has $2^{\mathrm{Ko}}$ direct summands in any decomposition into the direct sum of cyclic groups of order $p$. But, in the latter case, as the group is bound to be the direct sum of cyclic groups of order $p$, all what we have to prove is that the cardinal or the group is $\geqslant 2^{\mathrm{X}_{0}}$.

The proof splits into two cases:

1. If $\gamma_{p}^{(0)} \neq 0$, then $\gamma_{p}^{(0)} \geqslant 2^{\mathrm{K}_{0}}$.

Let $\alpha$ be the natural homomorphism

$$
\alpha: G \stackrel{\text { onto }}{\rightarrow} G / \operatorname{gp}\{H, p G\}
$$

LEMMA 1. Suppose that for any natural number $n$ there exists an element $x^{\prime}=x^{\prime}(n)$ in $G$ which is not representable as the sum of an element of order $\leqslant n$ and an element divisible by $p$. Then $S^{*}$ contains a subset $P$ such that $\overline{\overline{a \pi(P)}}=2^{\mathrm{X}_{0}}$, whence $\gamma_{p}^{(0)} \geqslant 2^{\mathrm{X}_{0}}$.

Proof of the lemma. Let $U^{(n)}$ denote the subset of the elements of $S^{*}$ whose orders are $\leqslant n$. Let $U=\bigcup_{n=1}^{\infty} U^{(n)}$. Since $S$ is pure in $S^{*}, \pi(U)$ $=H$. Let $H^{(n)}=\pi\left(U^{(n)}\right)$.

Under the assumption of the lemma we show that for any natural numbers $n, m$ there exists natu: al number $i=i(m, n)>m$ and an element $x_{i}$ in $G_{i}$ such that $x_{i}$ is not of the form $d+h$, where $d \epsilon p G_{i}$ and $h$ is of order $\leqslant n$.

To see this we note that, for any element $x=\left(x_{k}\right)_{k=1,2, \ldots .}$ in $\pi^{-1}\left(x^{\prime}(n !)\right)$, there is an infinite sequence $k_{j}, j=1,2, \ldots$, such that $x_{k j}$ is not the sum of an element of $p G_{k j}$ and an element of order $\leqslant n$.
In fact, in the contrary case, i.e. if for any $k$ greater than some $k_{0}$

$$
x_{k}=d_{k}+h_{k}
$$

where $d_{k} \in p G_{k}$ and the order of $h_{k}$ is $\leqslant n$, then for

$$
\begin{gathered}
\bar{x}_{k}=\left\{\begin{array}{cc}
x_{k} & \text { if } k>k_{0}, \\
\theta & \text { otherwise, }
\end{array} \quad \bar{d}_{k}= \begin{cases}d_{k} & \text { if } k>k_{0}, \\
\theta & \text { otherwise },\end{cases} \right. \\
\bar{h}_{k}= \begin{cases}h_{k} & \text { if } k>k_{0}, \\
\theta & \text { otherwise, }\end{cases}
\end{gathered}
$$

the elements $\bar{x}=\left(\bar{x}_{k}\right)_{k=1,2, \ldots}, \quad \bar{d}=\left(\bar{d}_{k}\right)_{k=1,2, \ldots}, \quad \bar{h}=\left(\bar{h}_{k}\right)_{k=1,2, \ldots}$ satisfy the relation

$$
x^{\prime}=\pi(x)=\pi(\bar{x})=\pi(\bar{d})+\pi(\bar{h}) .
$$

But this is impossible since $\bar{h}$ and so $\pi(\bar{h})$ has order at most $n$ ! and $\pi(\bar{d})$ is divisible by $p$.

We put $i(m, n)$ to be the first of the $k_{j}$ 's greater than $m$.

We define an infinite sequence $i_{n}, n=1,2, \ldots$, as follows: $i_{1}=i(1,1)$, $i_{n+1}=i\left(i_{n}, n !\right)$. For any infinite subsequence $\eta=\left\{i_{n r}\right\}, r=1,2, \ldots$, of $\left\{i_{n}\right\}, n=1,2, \ldots$, we define an element $x_{\eta}=\left(x_{k}\right)_{k=1,2, \ldots}$, where

$$
x_{k}= \begin{cases}x_{i_{n_{r}}} & \text { if } \quad k=i_{n_{r}}, \\ 0 & \text { otherwise. }\end{cases}
$$

It is clear that since $i_{n}, n=1,2, \ldots$, increases, the element $x_{\eta}$ is well defined. Let $P$ be the set of the elements $x_{\eta}$. We are going to prove that for any $\eta$ and an arbitrary choice of the ambigous sign \pm the element $\pi\left(\bar{x}_{\eta}\right)$, where $x_{\eta}=\left(x_{k}\right)_{k=1,2, \ldots}$ with

$$
\bar{x}_{k}= \begin{cases} \pm x_{k} & \text { for } k \epsilon \eta \\ 0 & \text { otherwise }\end{cases}
$$

is not representable as the sum of an element of $p G$ and an element of finite order. In fact, suppose to the contrary that

$$
\pi\left(\bar{x}_{\eta}\right)=d+h,
$$

where $d \in p G$ and $\lambda_{\epsilon} H^{(n)}$ for some $n$. Hence

$$
\bar{x}_{k}=d_{k}+h_{k}
$$

for all $k$ greater than $k_{0}$. But this fails to be true already for the first $k=i_{n_{r}}$ greater than $k_{0}$.

It is clear that $\overline{\overline{\pi(P)}}=2^{\mathrm{No}}$. Moreover, if $x_{\eta^{\prime}}$ and $x_{\eta^{\prime \prime}}$ are two elements of $P$ such that $\pi\left(x_{\eta^{\prime}}\right) \neq \pi\left(x_{\eta^{\prime \prime}}\right)$, then the sequences $\eta^{\prime}$ and $\eta^{\prime \prime}$ differ in an infinite sequence $\eta^{\prime \prime \prime}$ of indices, so

$$
x_{\eta^{\prime}}-x_{\eta^{\prime \prime}}=\left(y_{k}\right)_{k=1,2, \ldots} \quad \text { with } \quad y_{k}= \begin{cases} \pm x_{k} & \text { for } k \in \eta^{\prime \prime \prime}, \\ 0 & \text { otherwise. }\end{cases}
$$


Consequently, in virtue of what was proved above, $x_{\eta^{\prime}}-x_{\eta^{\prime \prime}}$ cannot be the sum of an element of finite order and an element of $p G$. Therefore it does not belong to the group $\operatorname{gp}\{H, p G\}$ and so $\alpha \pi\left(x_{\eta^{\prime}}\right)-\alpha \pi\left(x_{\eta^{\prime \prime}}\right) \neq 0$, which shows that $\alpha$ is one-to-one on $P$, whence $\alpha \pi(P)=2^{N_{0}}$. Lemma 1 is thus proved.

Now suppose that $\gamma_{p}^{(0)} \neq 0$, then, by $i^{\prime}, G$ contains an element which cannot be represented as the sum of an element divisible by $p$ and an element of finite order. Consequently, the conditions of lemma 1 are satisfied and so $G / g p\{H, p G\}>\alpha \pi(P)$, whence, by $\mathrm{i}^{\prime}, \gamma_{p}^{(0)} \geqslant 2^{\boldsymbol{k}_{0}}$.

To see that relation (a) holds we note that if $\gamma_{p}^{(n)}>0$ for infinitely many $n$, then $G$ contains direct summands $C_{p^{n}}$ for infinitely many $n$, therefore $G$ contains direct sumands being cyclic groups of order $n$, for infinitely many $n$, so for any natural number $n$ it contains an element $x^{\prime}(n)$ (the generator of $C_{p^{n}}$ ) which is not the sum of an element of order $\leqslant n$ and an element divisible by $p$. Hence, by lemma 1 and $\mathrm{i}^{\prime}, \gamma_{p}^{(0)} \geqslant 2^{\text {रo }}$.

2. If $\gamma_{p}^{(n)} \neq 0$ then $\gamma_{p}^{(n)} \geqslant 2^{\gamma_{0}}, n \geqslant 1$. Denote by $\alpha$ the natural homomorphism $\alpha:\left(p^{n-1} G\right)[p] \stackrel{\text { onto }}{\rightarrow}\left(p^{n-1} G\right)[p] /\left(p^{n} G\right)[p]$. Suppose $\gamma_{p}^{(n)} \geqslant 1$ and let $0 \neq \approx \epsilon\left(p^{n-1} G\right)[p] /\left(p^{n} G\right)[p]$.

Let, further, $y \in \alpha^{-1}(z)$. Then $y^{\prime}$ is an element of order $p$ belonging to $G$, so, since $S^{n}$ is pure in $S^{*}$, there exist an element $y=\left(y_{k}\right)_{k=1,2, \ldots}$ in $\pi^{-1}\left(y^{\prime}\right)$ whose order is $p$. Moreover, starting from some $k_{0}$ we have $y_{k} \in p^{n-1} G_{k}$ for $k>k_{0}$. Since $\pi(y) \neq 0$, there is an infinite sequence of different integers $k_{j}, j=1,2, \ldots$, such that $y_{k_{j}}$ is non divisible by $p^{n}$ in $G_{k j}$. Now we proceed as in case 1: for any infinite subsequence $\eta=\left\{k_{\gamma_{1}}\right\}, r=1,2, \ldots$, of $k_{j}, j=1,2, \ldots$, we define an element $x_{\eta}=\left(x_{k}\right), k=1,2, \ldots$, where

$$
x_{k}= \begin{cases}y_{k_{j_{r}}} & \text { if } k=k_{j_{r}}, \\ 0 & \text { otherwise. }\end{cases}
$$

It is also clear that, since for a $\eta$ there are infinitely many $x_{k}$ 's in $x_{\eta}=\left(x_{k}\right)$, $k=1,2, \ldots$, not divisible by $p^{n}$. Let $P_{n}$ be the set of the elements $x_{\eta}$. Clearly $\overline{\overline{\pi\left(P_{n}\right)}}=2^{x_{0}}$ and $\pi\left(P_{n}\right) \epsilon\left(p^{n-1} G\right)[p]$. So the same argument as in the case of $\gamma_{p}^{(0)}$ proves that $\alpha$ is one-to-one on $\pi\left(P_{n}\right)$, whence $\overline{\left(p^{n-1} G\right)[p] /\left(p^{n} G\right)}[p]$ $\geqslant \overline{a_{n} \pi\left(\overline{\left.P_{n}\right)}\right.}=2^{\mathrm{N}_{0}}$, what was to be proved.

In order to establish the theorem for $\delta$ and $\delta_{p}$ we prove

LEMTA 2. Suppose that

I. For any natural $n$ there is an element $x^{\prime}=x^{\prime}(n)$ in $G$ such that $x^{\prime}$ is divisible by $n$, then for any two natural numbers $m$, $n$, there exist a natural number $i=i(m, n)>m$ and an element $x_{i}$ in $G_{i}$ which is divisible by $n$.

If, in addition,

II. The order of $x^{\prime}$ is $\geqslant n$, or,

II'. the order of $x^{\prime}$ is equal to $p$,

then $i=i(m, n)$ and $x_{i}$ can be chosen in such a way that the order of $x^{i}$ is $\geqslant \pi$, or it is equal to $p$, respectively.
Proof. If $x=\left(x_{k}\right)_{k=1,2, \ldots} \in \pi^{-1}\left(x^{\prime}\right)$, then starting from some $k_{0}$ all $x_{k}$ with $k \geqslant k_{0}$ are divisible by $n$. So we take as $i(m, n)$ the first natural number greater than $m$ and not less than $k_{0}$, we put $x_{i}=x_{k}$ with $k=i(m, n)$.

If, in addition, the order of $x^{\prime}$ is $\geqslant n$, or alternatively, equal to $p$, then, in either case, there exists an infinite sequence $k_{j}, j=1,2, \ldots$, for which the order of $x_{k_{j}}, j=1,2, \ldots$, is $\geqslant n$ or equal to $p$, respectively. Thus for $i(m, n)$ we may take the first of the $k_{j}$ 's which is greater than $m$ and not less than $k_{0}$.

3. If $\delta \neq 0$, then $\delta \geqslant 2^{\aleph_{0}}$. It for any natural number $n$, the group $G$ contains an element $x^{\prime \prime}(n)$ of order $\geqslant n$, then conditions (I) and (II) of lemma 2 are satisfied. In fact, it is sufficient to put $x^{\prime}(n)=n x^{\prime \prime}\left(n^{2}\right)$. Hence it follows that if $\delta \geqslant 1$ or if any of conditions (c) is not valid, then the hypothesis of lemma 2 is satisfied. Thus the numbers $i=i(m, n)$ and the elements $x_{i}$, the orders of which are greater than $n$, respectively, exist. We define a sequence $i_{n}, n=1,2, \ldots$, by induction:

$$
i_{1}=i(1,1), \quad i_{n+1}=i\left(i_{n}, n !\right) \text {. }
$$

Let $\eta=\left\{i_{n_{r}}\right\}, r=1,2, \ldots$, be an infinite subsequence of $i_{n}$. We put

$$
x_{\eta}=\left(x_{k}\right)_{k=1,2, \ldots} \quad \text { where } \quad x_{k}= \begin{cases}x_{i_{n_{r}}} & \text { if } k=i_{n_{r}}, \\ 0 & \text { otherwise. }\end{cases}
$$

Clearly, the order of $\pi\left(x_{\eta}\right)$ is infinite and $\pi\left(x_{\eta}\right)$ is divisible by any natural number in $G$. We form the set $P$ consisting of the $x_{\eta}$ 's and we note that $\overline{\overline{\pi(}} P)=2^{\mathrm{N}_{0}}$.

Moreover, if $\alpha$ is the natural homomorphism of

$$
a: G \stackrel{\text { onto }}{\rightarrow} G / H
$$

where $H$ is the maximal periodic subgroup of $G$, then $\alpha$ is one-to-one on $\pi(P)$. (The argument for this purpose is almost the same as the one used in the proof of lemma 1.) From this we infer that $\overline{\alpha \pi(\bar{P})}=2^{\aleph_{0}}$, which means that $G$ contains $2^{N_{0}}$ elements, each divisible by any natural number, and not congruent to each other $\bmod H$. This, in view of the fact that the cardinal of the group of rationals is $s_{0}$, means that $2^{\boldsymbol{N}_{0}}$ groups of rationals must appear in any decomposition of the maximal divisible subgroup of $G$ into the direct sum of groups of rationals and Priufer's groups. Hence we see that

iii. If for any natural number $n$ the group $G$ contains an element $x^{\prime \prime}(n)$ of order $\geqslant n$, then $\delta=2^{N_{0}}$. of (c).

This proves the assertion about $\delta$ and, at.the same time, the validity 
4. If $\delta_{p} \neq 0$, then $\delta_{p} \geqslant 2^{\mathrm{x}_{0}}$. The proof for $\delta_{p}$ is almost the same as the one produced above for $\delta$. At first we note that either of the conditions $\delta_{p} \geqslant 1$ and

$$
\gamma_{p}^{(n)} \geqslant 1 \text { for infinitely many } n
$$

implies the validity of (I), (II) of lemma 2. Only the latter of the implications needs a proof. Suppose that (5) holds; then $G$ contains at least one pure cyclic subgroup of order $m_{n}, m_{1}<m_{2}<\ldots$ Let $x^{(n)}$ be the generator of the cyclic subgroup of order $m_{n}$. Then the element $x^{\prime}(n)=p^{m_{n}-1} x(n)$ satisfies $\mathrm{I}$ and $\mathrm{II}^{\prime}$ of lemma 2 . Now we show that

iv. Conditions I, $\mathrm{II}^{\prime}$ of lemma 2 imply $\delta_{p} \geqslant 2^{\mathrm{No}_{0}}$.

We take the $x_{i}$ 's such that the order of each of them is $p$. Then we form the set $P$ in analogy with previous case and we note that the elements of $\pi(P)$ are of order $p$ and, in addition, they are divisible by any natural number. From this we infer that $G$ contains a set of cardinal $2^{\gamma_{0}}$ consisting of elements of order $p$ divisible by any natural number. Since each of the $C_{p o}$ 's in any decomposition of the maximal divisible subgroup of $G$ into groups of rationals and Prüfer's groups $C_{p \infty}$ contains $p-1$ elements of order $p$. We see that $\delta_{p} \geqslant 2^{\mathrm{N}_{0}}$, and that condition (b) is satisfied.

THEOREM 2. Suppose that $\delta, \delta_{p}, \gamma_{p}^{(n)}, n=0,1,2, \ldots, p=2,3,5, \ldots$, is a family of cardinals, each equal to 0 or to $2^{\mathbf{x}_{0}}$, which satisfy conditions (a), (b), (c) of theorem 1. Then there exists a countable sequence of groups $G_{1}, G_{2}, \ldots$ with $\overline{\bar{G}}_{k} \leqslant 2^{\aleph_{0}}, k=1,2, \ldots$, such that if $S^{*} / S=G$, then $\delta=\delta(G)$, $\delta_{p}=\delta_{p}(G), \gamma_{p}^{(n)}=\gamma_{p}^{(n)}(G)$ for any $n=0,1,2, \ldots, p=2,3,5, \ldots$

Proof. We define a group $H$ as the direct sum of a family of groups which comprises one copy of each of the kinds of groups: groups of rationals $R$, Prüfer's groups $C_{p \infty}$ groups of $p$-adic integers $I_{p}$ and cyclic groups $C_{p^{n}}$ of order $n$ for which the corresponding cardinals $\delta, \delta_{p}, \gamma_{p}^{(0)}, \gamma_{p}^{(n)}$, $n=1,2, \ldots, p=2,3, \ldots$, are greater than zero. We put $G_{k}=H$ for all $k=1,2, \ldots$ and we form $S^{*}, S$ and $S^{*} / S=G$. We assert that

$$
\delta=\delta(G), \quad \delta_{p}=\delta_{p}(G), \quad \gamma_{p}^{(n)}=\gamma_{p}^{(n)}(G), \quad n=0,1,2, \ldots, p=2,3,5, \ldots
$$

Suppose that $\delta=0$, then, by (c) the group $H$ is the direct sum of finitely many of finite cyclic groups. Consequently $S^{*}$ is a group of finite exponent and so $S^{*} / S=G$ cannot contain any element of infinite order, whence $\delta(G)=0$.

If $\delta=2^{\aleph_{0}}$ the element $\left(x_{k}\right)_{k=1,2, \ldots} \in S^{*}$ with $x_{k} \in R \subset G_{k}$ has infinite order $\bmod S$ and is divisible by any natural number $n$, so, by iii, $\delta(G)=2^{\text {No. }}$.

Suppose that $\delta_{p}=0$, then, by (b), only finitely many of $\gamma_{p}^{(n)}, n=1$, $2, \ldots$, is different from zero, so the order of any element of $H$ which is a power of $p$ must be $\leqslant p$, where $m$ is the maximal natural number for which $\gamma_{p}^{(m)} \neq 0$, or zero if $\gamma_{p}^{(n)}=0$ for all $n$. Since $S$ is pure in $S^{*}$, any element of $G$ whose order is a power of $p$ must also be $\leqslant p^{m}$, so, clearly, $G$ cannot contain any $C_{p \infty}$, whence $\delta_{p}(G)=0$.

If $\delta_{p}=2^{x_{0}}$. The element $\left(x_{k}\right)_{k=1,2, \ldots} \in S^{*}$ with $x_{k} \in C_{p \infty} \subset G_{k}$ and $p x_{k}=0$ is mapped by $\pi$ onto an element $x$ of order $p$ divisible by any $n$, so by iv we have $\delta_{k}(G)=2^{N_{0}}$.

Suppose that $\gamma_{p}^{(0)}=0$. Then by (a) only finitely many of $\gamma_{p}^{(n)}$ with $n=1,2, \ldots$ are $\neq 0$. So $H=G_{k}=A_{k}+B_{k}, k=1,2, \ldots$, where $A_{k}$ consists of elements of order $\leqslant p^{m}, m$ being the greatest natural number for which $\gamma_{p}^{(n)} \neq 0$ and zero if $\gamma_{p}^{(n)}=0$ for all $n$, and any element of $B_{k}$ is divisible by $p$. Hence a similar decomposition is valid fo: $S^{*}$, whence $S^{*}=\operatorname{gp}\left\{U, p S^{*}\right\}$, where $U$ is the maximal periodic subgroup of $S$. Since $S$ is pure in $S^{*}$, this gives $G=S^{*} / S=\operatorname{gp}\{M, p G\}$, where $M$ is the maximal periodic subgroup of $G$. Hence, by $i^{\prime}$, we obtain $\gamma_{p}^{(0)}(G)=0$.

If $\gamma_{p}^{(0)}=2^{\boldsymbol{K}_{0}}$, then the element $\left(x_{k}\right)_{k=1,2, \ldots} \in S^{*}$, where $x_{k} \in G_{k}$ is an element not divisible by $p$ in $I_{p} \subset H=G_{k}$, is mapped by $\pi$ onto an element which is not the sum of an element of finite order and an element divisible by $p$, so, by lemma $1, \gamma_{p}^{(0)}(G)=2^{x_{0}}$.

Let $\gamma_{p}^{(n)}, n \geqslant 1$. Suppose that $\gamma_{p}^{(n)}=0$. Then $H$ does not contain any pure cyclic subgroup of order $p^{n}$, whence we infer that $S^{*}$ has the same property and so, since $S$ is pure in $S^{*}, G$ does not contain any pure cyclic subgroup of order $p^{n}$. This means that $\gamma_{p}^{(n)}(G)=0$.

If $\gamma_{p}^{(n)}=2^{N_{0}}$, then the element $\left(x_{k}\right)_{k=1,2, \ldots} \in S^{*}$ where $x_{k} \in G_{k}$ is the generator of the direct summand $C_{p^{n}}$ of $G_{k}$ is mapped by $\pi$ onto an element $x$ for which $p^{n-1} x \in\left(p^{n-1} G\right)[p]$ and $p^{n-1} x \notin\left(p^{n} G\right)[p]$, so $\left(p^{n-1} G\right)[p] /\left(p^{n} G\right)[p] \neq 0$, whence, by theorem $1, \gamma_{p}^{(n)}=2^{N_{0}}$.

\section{References}

[1] A. Hulanicki, The structure of the factor group of the unrestricted sum by the restricted sum of Abetian groups, Bull. Acad. Pol. Sci. Série sci. math., astr. et phys. 10 (1962), pp. 77-80.

[2] - Compact Abelian groups and extensions of Haar measures, Rozprawy Maematyczne 38 (1964).

[3] I. Kaplansky, Infinite Abelian groups, Ann Arbor 1954. [4] Л. Я. Куликов, Обобияедь
Общества 1 (1952), pp. 247-326.

INSTITUTE OF MATHEMATICS, THE UNIVERSITY, WROCEAW INSTYTUT MATEMATYCZNY, UNIWERSYTET, WROCEAW

INSTITUT OF MATHEMATICS OF THE POLISH ACADEMY OF SCIENCES INSTYTUT MATEMATYCZNY POLSKIEJ AKADEMII NAUK

Reģu par la Rédaction le 29. 8. 1962 